Article

\title{
VceC Mediated IRE1 Pathway and Inhibited CHOP-induced Apoptosis to Support Brucella Replication in Goat Trophoblast Cells
}

\author{
Feijie Zhi ${ }^{1,+}$, Dong Zhou ${ }^{1,2,+}$, Furong Bai ${ }^{1}$, Junmei Li ${ }^{1}$, Caixia Xiang ${ }^{1}$, Guangdong Zhang ${ }^{1}$, \\ Yaping Jin ${ }^{1,2}$ and Aihua Wang $1, *$ (D) \\ 1 Key Laboratory of Animal Biotechnology of the Ministry of Agriculture, Northwest A\&F University, \\ Yangling 712100, China \\ 2 College of Veterinary Medicine, Northwest A\&F University, Yangling 712100, China \\ * Correspondence: aihuawang@126.com \\ + These authors contributed equally to this work.
}

Received: 22 July 2019; Accepted: 20 August 2019; Published: 22 August 2019

\begin{abstract}
The effectors of the type IV secretion system (T4SS) of bacteria play important roles in mediating bacterial intracellular proliferation and manipulating host-related pathway responses to bacterial infection. Brucella Spp. inhibit the apoptosis of host cells to benefit their own intracellular proliferation. However, the underlying mechanisms between T4SS effectors and Brucella-inhibited apoptosis in goat trophoblast cells remain unclear. Here, based on Brucella suis vaccine strain 2, the $\mathrm{VceC}$ was deleted by allelic exchange. We show that $\Delta \mathrm{VceC}$ was able to infect and proliferate to high titers in goat trophoblast cells (GTCs) and increase C/EBP-homologous protein (CHOP)-mediated apoptosis. GRP78 expression decreased upon $\triangle \mathrm{VceC}$ infection. In addition, we discovered that the inositolrequiring enzyme 1 (IRE1) pathway was inhibited in this process. Changing endoplasmic reticulum (ER) stress affected Brucella intracellular replication in GTCs. The replication of $\Delta$ VceC was more sensitive under the different ERstress conditions in the GTC line after treatment with ER stress inhibitors 4 phenyl butyric acid (4-PBA) or ER stress activator Tm. Together, our findings show that $\mathrm{VceC}$ has a protective effect on the intracellular persistence of Brucella infection, and inhibits ER stress-induced apoptosis in the CHOP pathway. The present work provides new insights for understanding the mechanism of $\mathrm{VceC}$ in the establishment of chronic Brucella infection.
\end{abstract}

Keywords: Brucella suis S2; type IV secretion system; VceC; goat trophoblast cells; apoptosis; endoplasmic reticulum stress; unfold protein response

\section{Introduction}

Brucellosis is a zoonotic infectious disease caused by bacteria of the genus Brucella. The infection affects more than 500,000 people across the world, a number that may be higher in agricultural communities worldwide [1,2]. Brucella, a Gram-negative and facultative intracellular bacterium, mainly parasitizes in phagocytic cells, such as macrophages and trophoblast cells [3,4]. The pathogenicity of Brucella is due to its ability to adapt to the environmental conditions encountered in its intracellular replicative niche including low levels of nutrients and oxygen, acidic $\mathrm{pH}$, and reactive oxygen intermediates [5]. To date, no vaccine can be safely and effectively used to prevent human brucellosis, and the disease in human is difficult to treat with antibiotics [6]. Because of the Brucella characteristics, it could be used as a bioweapon [7]. The Brucella vaccines, strain 19 and RB51, are effective in controlling brucellosis in animal [8]. Brucella suis vaccine strain 2 (B. suis S2), a naturally attenuated variant in china, was isolated from the embryo of an aborted sow in 1952 by the researchers of 
China Institute of Veterinary Drug Control and is most extensively used for the prevention and control of brucellosis in sheep, goats, cattle, and other domestic animals [9]. However, these vaccines have numerous drawbacks, including interference with diagnostic tests, pathogenicity for humans, potential to cause abortion in pregnant animals, and so on. Therefore, it is critical to understand the molecular mechanisms of Brucella intracellular survival and proliferation during infection for preventing brucellosis and developing vaccines.

The type IV secretion system (T4SS) is essential for persistent Brucella infection, since T4SS mutants are incapable of intracellular survival and replication in phagocytic cells and attenuated in a mouse model of infection [10]. T4SS injects Brucella effector proteins from the bacterium into the host-cell cytosol to impact cellular homeostasis and normal physiology. De Jong et al. [11] provide first direct evidence that effector protein VceC is conserved in all sequenced Brucella genomes and is translocated into cells by the Brucella T4SS. The translocated VceC results in a cytotoxic effect on macrophages. VceC translocates to the endoplasmic reticulum (ER) where it binds the GRP78 and induces an IRE1 $\alpha$-dependent inflammation [12]. Keestra-Gounder et al. [13] suggest that VceC can trigger ER stress, contributing to abortion during B. abortus infection in mice.

In animal primary hosts, Brucella have a particular tropism for the reproductive system, often leading to abortion in pregnant female animals. Because of the presence of high Brucella loads within placental trophoblast cells, the infection ultimately results in disruption of the placenta and infection of the fetus [14]. Therefore, trophoblast cells are a primary cellular target for the efficient survival and proliferation of Brucella in the natural host. However, the molecular mechanisms of the Brucella infectious process in goat trophoblast cells (GTCs) remain unclear. Once inside the host cells, Brucella in turn interact with the early and late endosomes, ER, and autophagy-like vacuoles, resulting in the completion of the intracellular lifecycle of Brucella and cell-to-cell spreading [4,5]. Brucella require fusion with the ER for survival, establishing a proliferation niche, and multiplication within host cells [15]. The ER fusion dramatically restructures the ER and disrupts ER homeostasis, leading to a condition known as ER stress [16]. To maintain ER homeostasis, the unfolded protein response (UPR) is induced, especially by the inositol-requiring enzyme 1 (IRE1) pathway, which promotes Brucella intracellular survival and proliferation in macrophages or GTCs $[13,17]$. In response to ER stress, the binding immunoglobulin protein (Bip, also known as GRP78) is recruited away from three sensors that are located in the ER membrane to assist in refolding proteins within the ER, resulting in activation of the UPR signaling pathway [18]. However, when persistent or excessive ER stress exceeds the ability of the UPR to manage misfolded and unfolded proteins, the UPR switches from an adaptive pathway to one that induces cell death [18]. UPR-mediated apoptosis is a new apoptosis signaling pathway, and one of the most significant activations of this pathway is induced by CHOP [19]. The manipulation of host cell death is a critical strategy of Brucella to maintain dissemination and intracellular persistence. The VceC also mediates the cytotoxicity effect by translocation of this effector protein into macrophages resulting in lysis of the host cells [11]. However, the interaction of $\mathrm{VceC}$ and B. suis S2-induced apoptosis mediated by ER stress in GTCs has scarcely been studied.

Here, we constructed a B. suis S2 T4SS effector protein VceC deletion mutant and investigated the mechanisms of VceC on B. suis S2-induced apoptosis in GTCs. Our results showed that the VceC mutant increased CHOP expression, interacted with GRP78, and mediated IER1 pathway of UPR to promoted apoptosis in GTCs. The replication of the VceC mutant was more sensitive under the different ER stress conditions after treatment with ER stress inhibitors or inducers in the GTC line. These findings demonstrate that $\mathrm{VceC}$ is a vital Brucella virulence by activating ER stress and further manipulating UPR to inhibit GTC apoptosis during Brucella infection. 


\section{Results}

\subsection{Compare to VceC Amino Acid Sequences Derived from Different Brucella Strains}

VceC is present in all sequenced Brucella strains, including B. suis S2 (BSS2_I1011), B. suis 1330 (BR1038), B. abortus 2308 (BAB1_1058), B. melitensis 16M (BEMI0948), and B. cains GB1 (C6Y57_05925) (Figure 1). VceC amino acid sequences were compared and analyzed based on the above strains. The VceC protein of B. suis S2 contained 410 amino acids, with a proline rich central domain. An N-terminal region of approximately 260 amino acids was conserved in proteins of the above strains. A 1 bp missed in B. suis and B. cains GB1 vceC led to a frameshift in the C-termini of B. suis and B. canis GB1. VceC protein had one amino acid change (Ser-275) in B. abortus 2308, one amino acid change (Asn-343) in B. cains GB1, and two amino acid changes (Phe-264 and Ser-275) in B. melitensis 16M, when compared with B. suis $\mathrm{S} 2$.

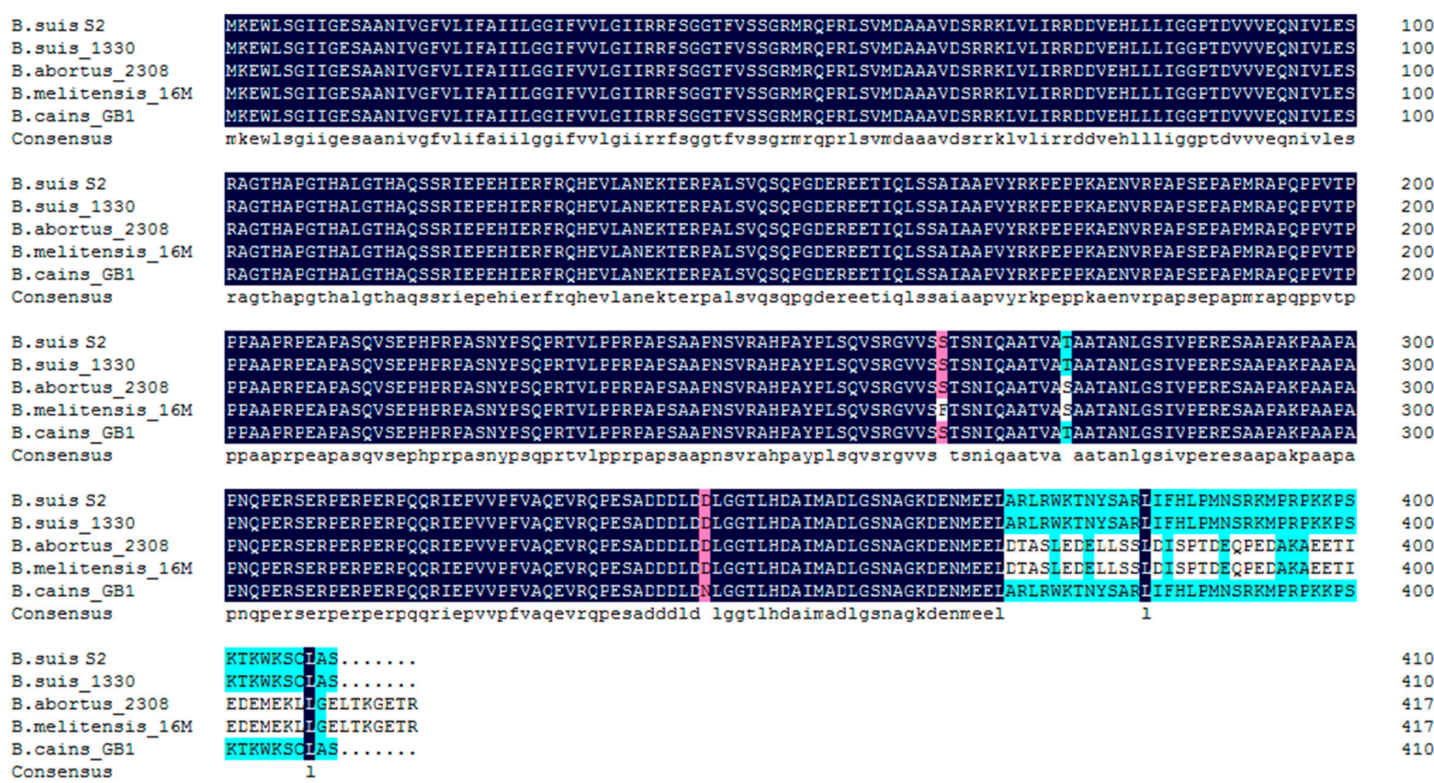

Figure 1. Alignment of VceC nucleotide sequences (A) and amino acid sequences (B) for Brucella suis S2 (BSS2_I1011), B. suis 1330 (BR1038), B. abortus 2308 (BAB1_1058), B. melitensis 16M (BEMI0948), and B. cains GB1 (C6Y57_05925). Amino acid differences are shaded in red and green.

\subsection{Mutant Strains $\Delta$ VceC Intracellular Survival in GTCs}

The VceC gene deletion mutant based on B. suis S2 was successfully constructed. Trophoblast cells were a primary cellular target for Brucella in the natural host. Our previous study demonstrated B. suis S2 was able to infect GTCs cultured in vitro [17] To evaluate the bacterial adherence and intracellular survival in GTCs, the number of CFUs was counted following infection with B. suis S2 and the mutant strains $\Delta \mathrm{VceC}$ at 100 multiplicity of infection (MOI). The bacterial adherence of $\Delta \mathrm{VceC}$ was not significantly different compared to B. suis S2 strains (data not shown). We demonstrated B. suis S2 (Figure 2A) and $\Delta \mathrm{VceC}$ (Figure $2 \mathrm{~B}$ ) were able to infect GTCs cultured in vitro. To determine whether deletion of VceC affected the intracellular survival of Brucella, the number of CFUs was counted following infection with $B$. suis $\mathrm{S} 2$ and $\Delta \mathrm{VceC}$ at different times. The results indicated the intracellular survival of $B$. suis $S 2$ and $\Delta \mathrm{VceC}$ occurred in a time-dependent manner. However, the bacterial intracellular survival of $\Delta \mathrm{VceC}$ was not significantly different (Figure $2 \mathrm{C}$ ). 


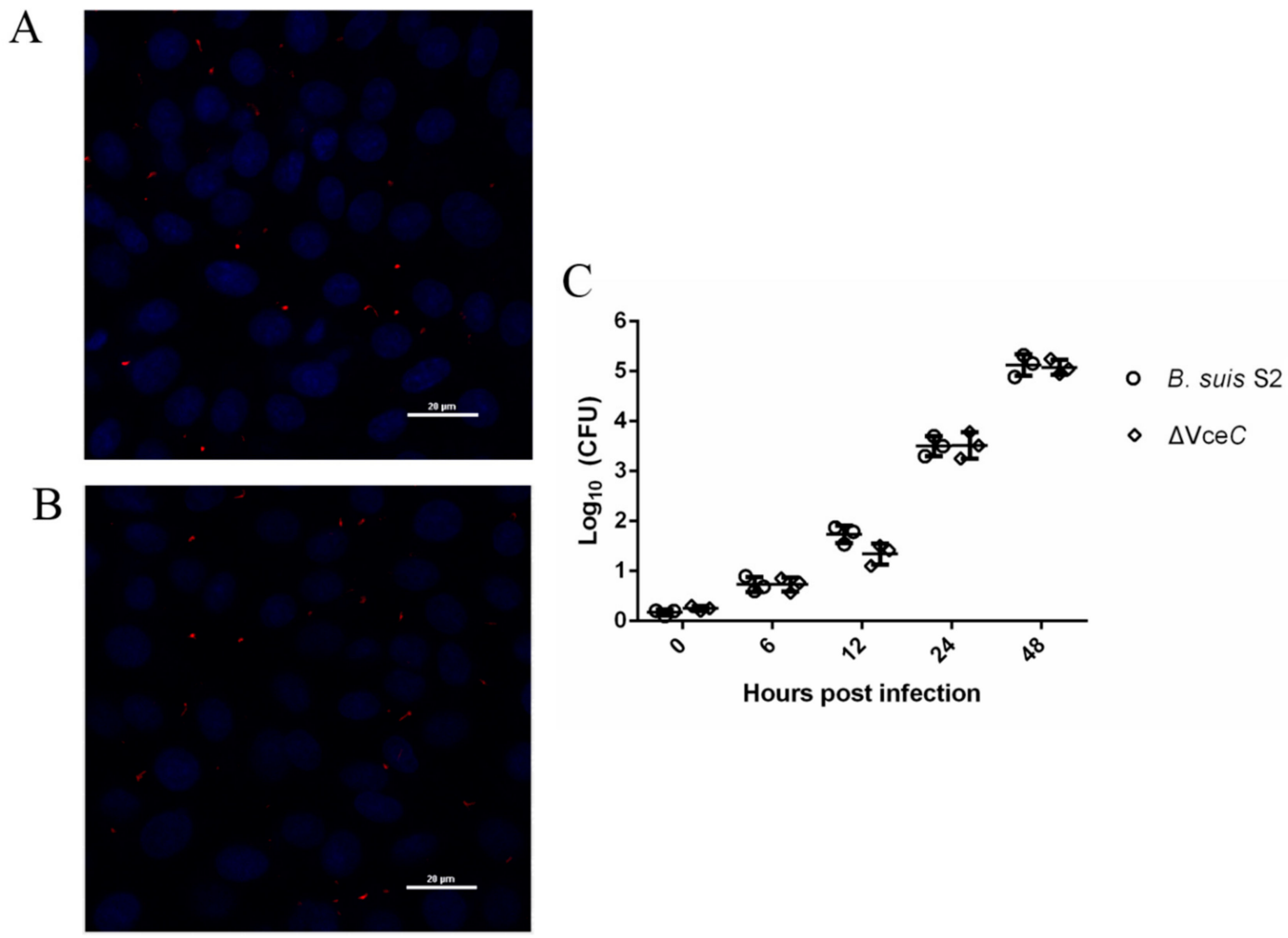

Figure 2. Infection and proliferation of B. suis $\mathrm{S} 2$ and $\Delta \mathrm{VceC}$ in goat trophoblast cells (GTCs). (A,B) Representative confocal micrographs of GTCs infected with B. suis S2 (A, red) and $\Delta \mathrm{VceC}(\mathbf{B}$, red) at $24 \mathrm{~h}$ post infection. The data shown are representative of three independent experiments. (C) Intracellular survival in GTCs of wild type (B. suis S2, circle) and the mutant obtained ( $\Delta \mathrm{VceC}$, square). The results are expressed as the means \pm standard deviation from three independent experiments at each time point.

\subsection{Effect of $\triangle V c e C$ on $G T C$ Apoptosis}

To evaluate whether the deletion of VceC affected the apoptosis level of GTCs infected by B. suis S2, we assessed the effect of apoptosis by flow cytometry in combination with Annexin V/PI double staining. When the cells were infected with $\Delta \mathrm{VceC}$ for $12 \mathrm{~h}$ and $48 \mathrm{~h}$, the average apoptosis of only Annexin V-positive cells (early apoptosis cells) increased significantly, reaching approximately $3.04 \pm 0.10 \%$ and $8.96 \pm 0.99 \%$, respectively (Tables 1 and 2 and Supplementary Figure S1). However, the average apoptosis of early apoptosis cells to $B$. suis S2 was approximately $1.77 \pm 0.23 \%$ and $5.15 \pm 2.46 \%$, respectively (Tables 1 and 2 and Supplementary Figure S1). The results demonstrated that $\Delta \mathrm{VceC}$ increased the proportion of early apoptosis in GTCs at $12 \mathrm{~h}$ and $48 \mathrm{~h}$ post infection.

Because $\Delta \mathrm{VceC}$ infection increased cells' early apoptosis, we measured cleaved caspase-3 (the marker of apoptosis), procaspase-9 (the marker of mitochondrion-induced cell death), and CHOP (the marker of ER stress-induced cell death) expression by Western blot at different times (Figure 3). At $48 \mathrm{~h}$, cleaved caspase-3 expression was enhanced after $\Delta \mathrm{VceC}$ infection compared with the B. suis S2 infected group. However, cleaved caspase- 3 expression was not significantly different at 0,12 , and $24 \mathrm{~h}$ among the uninfected group, $\Delta \mathrm{VceC}$-infected group, and B. suis S2-infected group. Caspase-9 expression was also not significantly different at $0,12,24$, and $48 \mathrm{~h}$ among the uninfected group, $\triangle \mathrm{VceC}$-infected group, and B. suis $\mathrm{S} 2$ infection group. Furthermore, $\mathrm{CHOP}$ expression was increased at 24 and $48 \mathrm{~h}$ after $\Delta \mathrm{VceC}$ infection compared with the B. suis $\mathrm{S} 2$ infection group, but no significant difference was found at 0 and $12 \mathrm{~h}$. According to our Western blot analysis, cleaved caspase-3 expression at $48 \mathrm{~h}$, as well as CHOP expression at $24 \mathrm{~h}$ and $48 \mathrm{~h}$, was more strongly induced in the $\Delta \mathrm{VceC}$ infection group than in the B. suis S2 infection group. The results suggested $\mathrm{VceC}$ is involved in Brucella-mediated apoptosis through the UPR signaling pathway. 
Table 1. Results of Annexin V-FITC/PI staining for cell apoptosis after B. suis S2 and $\Delta$ VceC infection at $12 \mathrm{~h}$.

\begin{tabular}{cccc}
\hline Group & Normal Cells & $\begin{array}{c}\text { The Early Apoptotic Cells } \\
\text { (\%) }\end{array}$ & $\begin{array}{c}\text { The Late Apoptotic Cells } \\
\text { (\%) }\end{array}$ \\
\hline Control & $85.05 \pm 0.63$ & $1.77 \pm 0.23$ & $3.59 \pm 0.25$ \\
B. suis S2-infected & $84.45 \pm 4.03$ & $1.23 \pm 0.34$ & $3.96 \pm 0.49$ \\
$\Delta$ VceC-infected & $84.55 \pm 1.06$ & $3.04 \pm 0.10^{*}$ & $4.08 \pm 0.74$ \\
\hline
\end{tabular}

Data represent the means \pm standard deviations from three replicates. The asterisks ${ }^{*}$ ) represent significant differences $(p<0.05)$ of the cell cycle distribution in GTCs infected by $\Delta$ VceC compared to that in B. suis S2-infected cells.

Table 2. Results of Annexin V-FITC/PI staining for cell apoptosis after B. suis S2 and $\Delta$ VceC infection at $48 \mathrm{~h}$.

\begin{tabular}{cccc}
\hline Group & Normal Cells & $\begin{array}{c}\text { The Early Apoptotic Cells } \\
\mathbf{( \% )}\end{array}$ & $\begin{array}{c}\text { The Late Apoptotic Cells } \\
\text { (\%) }\end{array}$ \\
\hline Control & $75.20 \pm 1.41$ & $5.15 \pm 2.46$ & $8.01 \pm 4.51$ \\
B. suis S2-infected & $79.50 \pm 3.68$ & $5.25 \pm 0.71$ & $6.18 \pm 0.51$ \\
$\Delta$ VceC-infected & $72.85 \pm 2.47$ & $8.96 \pm 0.99 *$ & $6.37 \pm 0.98$ \\
\hline
\end{tabular}

Data represent the means \pm standard deviations from three replicates. The asterisks $\left(^{*}\right)$ represent significant differences $(p<0.05)$ of the cell cycle distribution in GTCs infected by $\Delta \mathrm{VceC}$ compared to that in B. suis S2-infected cells.

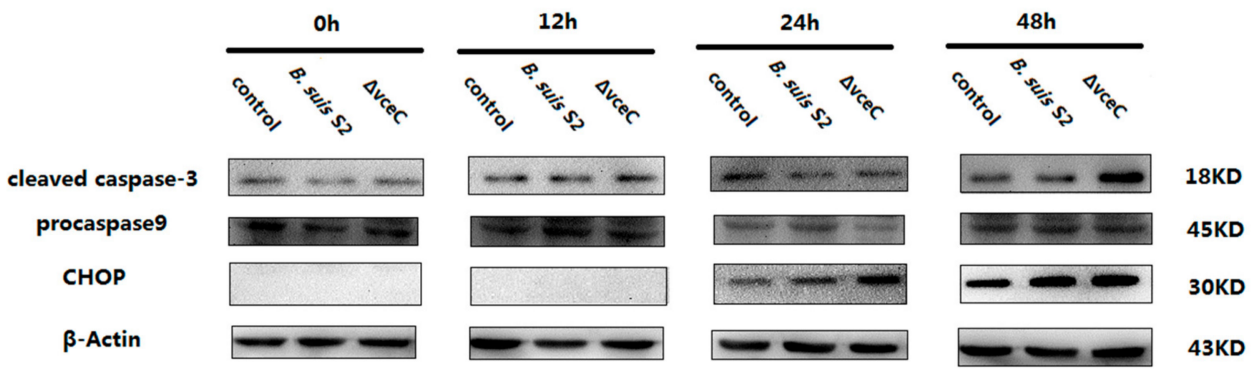

Figure 3. Effect of deletion of VceC on apoptosis of Brucella following infection of GTCs. GTCs were infected with $100 \mathrm{MOI}$ of $B$. suis S2 and $\Delta \mathrm{VceC}$ for $0 \mathrm{~h}, 12 \mathrm{~h}, 24 \mathrm{~h}$, and $48 \mathrm{~h}$, lysed, and subjected to Western blot analysis to detect the expression of apoptosis-related cleaved caspase-3, caspase-9, and $\mathrm{CHOP}$ proteins. The data shown are representative of three independent experiments.

\subsection{Deletion of VceC Decreases Brucella-Mediated ER Stress}

UPR is a cytoprotective response that is aimed at monitoring the survival and proliferation of intracellular pathogens. To more directly examine ER stress after $\Delta \mathrm{VceC}$ infection in GTCs, we examined the expression of GRP78 in $\triangle \mathrm{VceC}$ infection GTCs. GRP78 expression was increased at 24 and $48 \mathrm{~h}$ in the Brucella-infected group compared to the uninfected group and was decreased at 24 and $48 \mathrm{~h}$ in the $\Delta \mathrm{VceC}$-infected group compared to the Brucella-infected group, but no significant difference was found at 0 and $12 \mathrm{~h}$ among all groups (Figure $4 \mathrm{~A}-\mathrm{C}$ ). The results indicate the deletion of VceC changes Brucella-mediated ER stress.

To investigate UPR induction during $\triangle \mathrm{VceC}$ infection, GTCs were infected with $B$. suis $\mathrm{S} 2$ or $\Delta \mathrm{VceC}$, and the activation of three UPR sensors was analyzed by Western blot. ATF6- $\alpha$, ATF6- $\beta$, and phosphoeIF- $2 \alpha$ (downstream protein of PERK branch) were not significantly different in all groups (Figure 4D). However, PhosphoIRE1 expression was decreased at 12, 24, and $48 \mathrm{~h}$ in the $\Delta \mathrm{V}$ ceC infection group compared to the Brucella infection group (Figure 4D). We conclude that $\mathrm{VceC}$ is involved with Brucella-mediated ER stress by IRE1 branch activation of UPR. 
A

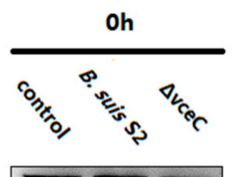

GRP78

CHOP

$\beta$-Actin

B

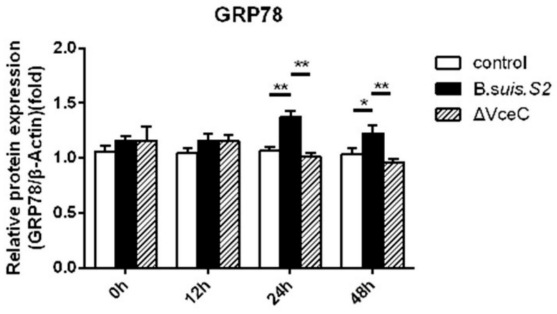

$12 \mathrm{~h}$

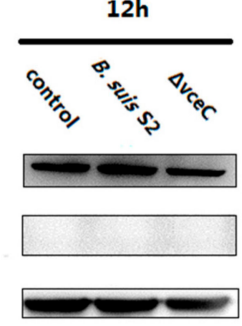

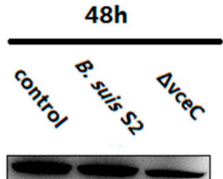

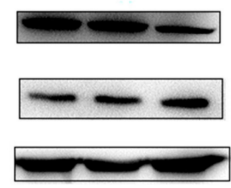

78KD

30KD

43KD

C

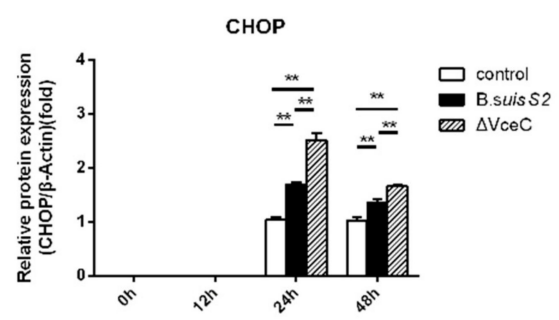

D
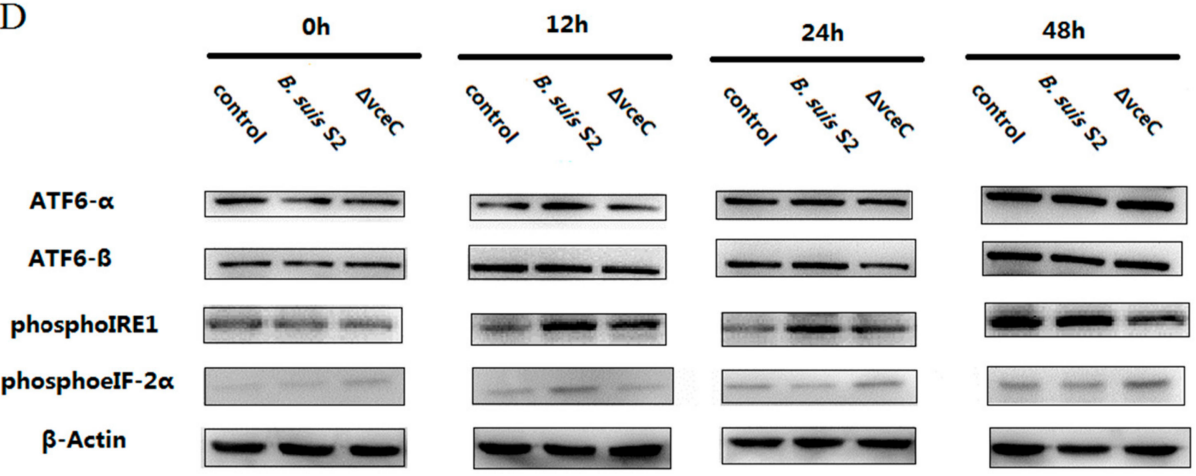

90KD

110KD

110KD

37KD

43KD

Figure 4. The unfolded protein response (UPR) pathway is induced by B. suis S2 and $\Delta$ VceC. (A) GTCs were infected with $100 \mathrm{MOI}$ of $B$. suis S2 and $\Delta \mathrm{VceC}$ for $0 \mathrm{~h}, 12 \mathrm{~h}, 24 \mathrm{~h}$, and $48 \mathrm{~h}$, and the untreated group was used as a negative control, followed by lysis and detection of GRP78 and CHOP protein expression by Western blot. The data shown are representative of three independent experiments. (B,C) Quantification of band intensities from three independent results was determined by densitometric analysis. Data represent the mean \pm standard deviation from three independent experiments at each time point. ${ }^{*} p<0.05 ;{ }^{* *} p<0.01$ (D) GTCs were infected with $100 \mathrm{MOI}$ of $B$. suis S2 and $\Delta$ VceC for 0,12 , 24 , and $48 \mathrm{~h}$, and the untreated group was used as a negative control, followed by lysis and detection of ATF6- $\alpha$, ATF6- $\beta$, phosphoIRE1, and phosphoeIF- $2 \alpha$ protein expression by Western blot. The data shown are representative of three independent experiments.

\subsection{The Replication of $\triangle V c e C$ Was More Sensitive after Changing ER Stress in GTCs}

To more directly assess the role of ER stress in Brucella survival and proliferation in GTCs, we explored whether ER stress altered by Tm or 4-PBA affects B. suis S2 or $\triangle$ VceC in GTCs. Increasing ER stress with $0.5 \mu \mathrm{g} / \mathrm{mL}$ Tm significantly increased ER stress marker GRP78 and CHOP expression (Figure 5A,B). In contrast, decreasing ER stress with $1 \mu \mathrm{M}$ 4-PBA (Figure 5A,B) significantly inhibited GRP78 and CHOP protein expression. In addition, increasing ER stress with Tm significantly inhibited the proliferation of B. suis $\mathrm{S} 2$ or $\triangle \mathrm{VceC}$, and decreasing ER stress with 4-PBA enhanced the proliferation of B. suis S2 or $\Delta \mathrm{VceC}$ (Figure $5 \mathrm{~B}-\mathrm{E}$ ). This observation is consistent with other reports that Brucella proliferation in host cells is affected by apoptosis in infected cells. Furthermore, the proliferation of $\triangle \mathrm{VceC}$ was significantly different compared with that of the B. suis S2 infection group under changing ER stress. The results reconfirmed that the deletion of VceC changes Brucella-mediated ER stress. 

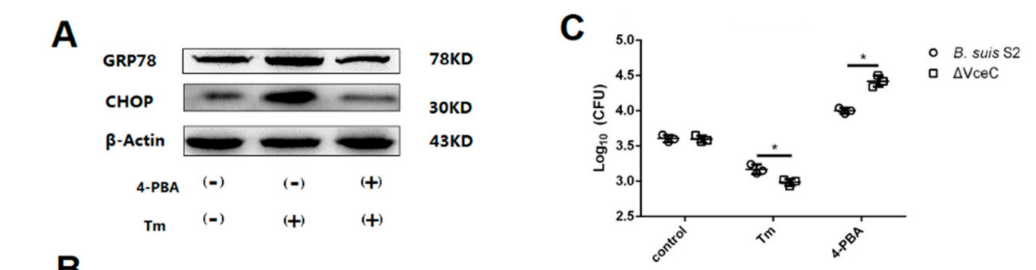

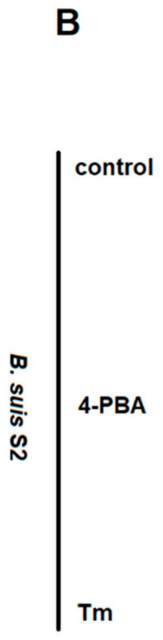
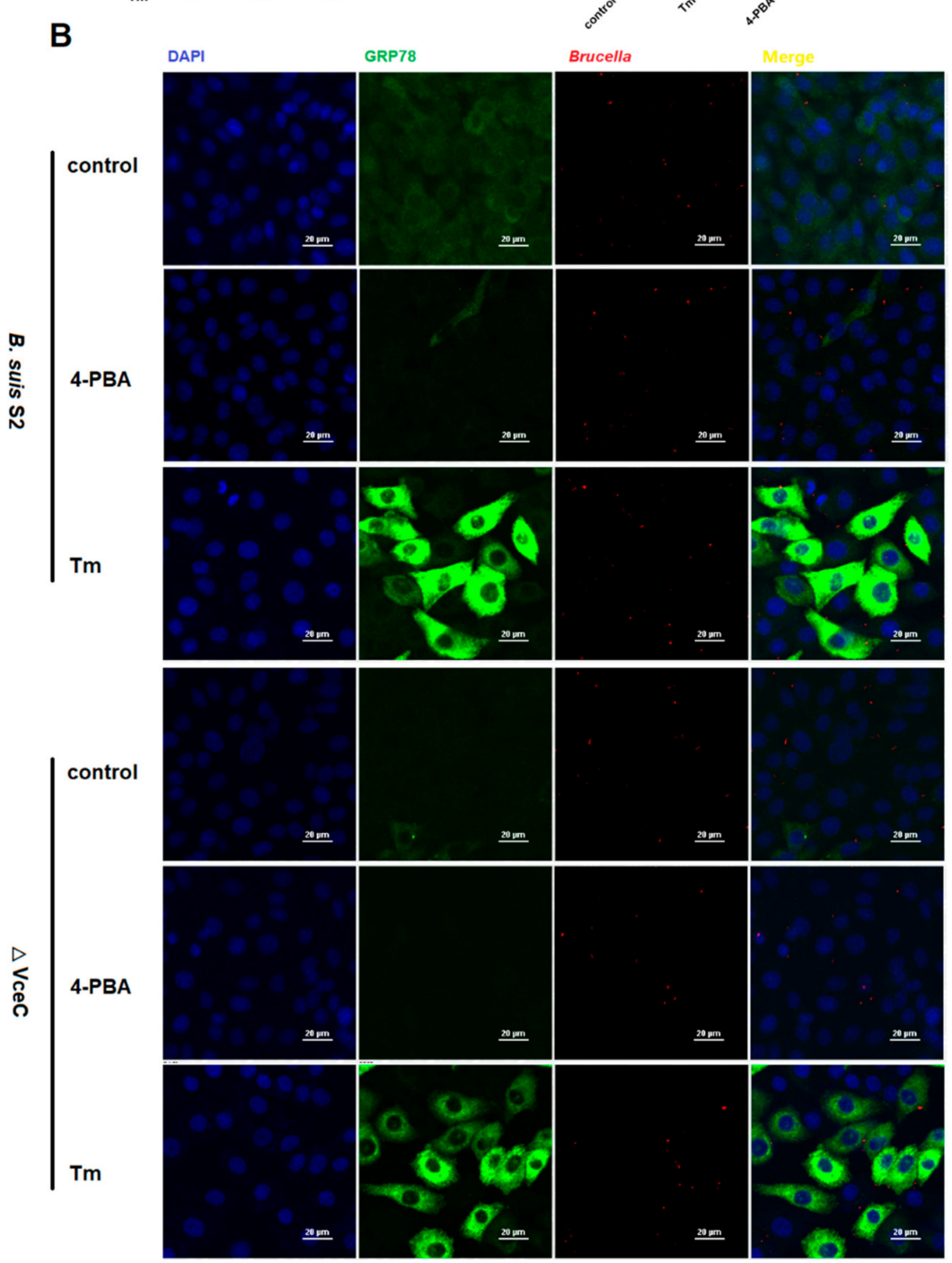

Figure 5. Intracellular survival of $B$. suis $\mathrm{S} 2$ and $\Delta \mathrm{VceC}$ by changing endoplasmic reticulum (ER) stress. (A) The UPR marker GRP78 and CHOP were analyzed using Western blot by treating with $0.5 \mu \mathrm{g} / \mathrm{mL}$ Tm or $1 \mu \mathrm{M} 4$-PBA in GTCs to establish an ER stress activated and inhibited model. The data shown are representative of three independent experiments. (B) (bar $=20 \mu \mathrm{m}$ ) Representative confocal micrographs of GRP78 protein (green) infected with B. suis S2 (red) and $\Delta \mathrm{VceC}$ (red) by added $0.5 \mu \mathrm{g} / \mathrm{mL}$ Tm or $1 \mu \mathrm{M}$ 4-PBA before infection in GTCs. The blue represents cell nucleus. (D-E) CFUs of B. suis S2 (C) and $\Delta \mathrm{VceC}(\mathrm{D})$ were determined in GTCs at $24 \mathrm{~h}$ post infection. The $0.5 \mu \mathrm{g} / \mathrm{mL}$ Tm or $1 \mu \mathrm{M} 4$-PBA was added before infection. Data represent the mean \pm standard deviation from three independent experiments. ${ }^{*} p<0.05$

\section{Discussion}

The ER mediates biosynthesis, folding, and modification of secretory and transmembrane proteins as well as maintains of calcium homeostasis. Elevated physiological demand for protein folding can disrupt the ability of ER, leading to misfolded or unfolded protein accumulation in this 
organelle, a condition called ER stress. The unfolded protein response (UPR) is induced to restore ER homeostasis [18]. However, excessive stress to the ER triggers CHOP-induced apoptosis [20].

Our results indicated that the B. suis S2 T4SS-secreted protein VceC can trigger ER stress but inhibit CHOP-induced apoptosis. The manipulation of host cell death is a critical strategy of Brucella to maintain dissemination or long-term intracellular persistence $[5,21]$. Macrophage/monocytes infected with Brucella suis or Brucella melitensis stains inhibit the apoptosis pathways, whereas GTCs infected with B. suis S2 undergo apoptotic cell death mediated by ER stress $[17,22,23]$. T4SS is one of the key factors for Brucella intracellular survival and plays an essential role in the inhibition of host-cell death [24]. Inactivation of virB, which encodes the T4SS, in smooth B. melitensis prevented the cytotoxicity of Brucella for macrophages; in contrast, overexpression of virB enhances cytotoxic effects [24]. The VceC also mediates the cytotoxicity effect by translocation of this effector protein into macrophages, resulting in lysis of the host cells [11]. However, the cytotoxicity or lysis of Brucella for host cells resembles oncosis and nerosis, but not apoptosis [11]. In our studies, B. suis S2 infection induced caspase-9 protein expression in GTCs, but, interestingly, VceC mutants enhanced cleaved caspase-3 and CHOP protein expression and induced an increase in apoptosis in trophoblast cells. During ER stress, CHOP expression increases to activate downstream genes, leading to ER stress-induced apoptosis $[25,26]$. Martinon et al. [27] suggested that Brucella lipopolysaccharide (LPS) may sufficiently temper CHOP induction to avert apoptosis. These findings suggested that VceC may synergize with LPS to inhibit CHOP-induced apoptosis. Further work is needed to verify the interaction between VceC and LPS during Brucella infection. Therefore, our results demonstrated that $\mathrm{VceC}$ promoted intracellular persistence of Brucella infection, which may be related to decreased CHOP expression and inhibition of the ERS-induced apoptosis pathway.

UPR is known to support the Brucella intracellular life cycle within the host cell. First, the UPR mobilizes amino acid transport and supports lipid biogenesis. Second, the UPR regulates autophagy to providing more nutrients. Third, the UPR enhances the protein-folding capacity to suppress downstream apoptosis. Fourth, the UPR allows cells to cope with oxidative stresses. Finally, the UPR enables host cells to survive the disruption of the ER structure and function [16]. During B. abortus infection, the effector protein VceC interacts with the ER chaperone GRP78 and localizes to the ER, then disrupts the ER structure, and results in ER stress in HeLa cells [12]. Our previous study shows that inhibiting ER stress with 4-PBA increased the number of B. suis S2 CFUs in GTCs. Enhancing ER stress with Tm inhibited the proliferation of B. suis S2 in GTCs [17]. GRP78 plays an essential role in supporting Brucella replication in GTCs. Decreasing GRP78 expression inhibited B. suis S2 proliferation in GTCs by promoting ER stress-induced apoptosis [17]. Those findings are consistent with our result that the $\mathrm{VceC}$ mutant reduced the GRP78 expression and enhanced that of CHOP to promote ER stress-induced apoptosis. The replication of the VceC mutant was more sensitive under the different ER stress conditions in the GTC line after treatment with ER stress inhibitors 4-PBA or ER stress inducer Tm. Our studies indicate that effector protein VceC is essential for Brucella replication by interaction with GRP78 to inhibit the apoptosis of B. suis S2-infected GTCs.

ER stress transmembrane sensor IRE1 plays a pivotal role in Brucella replication. Previous studies suggested that $B$. abortus infection of macrophages or HeLa cells activates IRE1 $\alpha$ pathway $[16,28]$. Yip1A, a host protein, is required for $\mathrm{rBCV}$ biogenesis and intracellular B. abortus replication through mediated IRE1 activation in HeLa cells [28]. De Jong et al. [12] demonstrated that IRE1-mediated UPR activation is activated by VceC. In our study, we confirmed that VceC mutant infection did not activate the IRE1 pathway in GTCs. VceC mutant infection did not affect the B. suis S2 CFU in GTCs. Our results are consistent with the study that IRE1 knockdown in bone marrow-derived macrophages (BMDM) or decreasing phosphoIRE1 $\alpha$ and IRE1 $\alpha$ in GTCs did not affect the number of Brucella bacteria at $24 \mathrm{~h}$ post infection $[17,28]$. An in vivo study suggested that infection with the B. abortus wild type and the VceC mutant resulted in similar numbers in the placenta of mice [13]. Taken together, we speculated that more effector protein synergies exist with VceC to activate the IRE1 pathway of UPR to support 
the Brucella replication in host cells, since inactivation of $\mathrm{VceC}$ alone did not affect the replication competence of Brucella [12].

In summary, the results demonstrated that B. suis S2 T4SS effector protein VceC decreased CHOP expression, interacted with GRP78 to inhibit apoptosis, and mediated the IER1 pathway of UPR to support B. suis S2 replication in GTCs. Our findings also suggested that more effector proteins should exist in synergy with $\mathrm{VceC}$ to support persistent Brucella intracellular infection. The present work provides new insights for understanding the mechanism of $\mathrm{VceC}$ in the establishment of chronic Brucella infection.

\section{Materials and Methods}

\subsection{Bacterial Strains}

The bacterial strains used in this study were smooth attenuated virulent Brucella suis vaccine strain 2 (B. suis S2, CVCC70502), and they were obtained from the Chinese veterinary culture collection center (Beijing, China). The B. suis S2 were grown in tryptic soy broth (TSB; Takana) and tryptic soy ager (TSA; Takana). Goat trophoblast cells (GTCs) were immortalized by transfection with human telomerase reverse transcriptase (hTERT); these cells were provided by Dewen Tong (Northwest A\&F University, Yangling, Shaanxi, China). For infection of GTCs, the bacteria were collected by centrifugation at $6000 \times \mathrm{g}$ for $10 \mathrm{~min}$ at $4{ }^{\circ} \mathrm{C}$ and washed three times with $15 \mathrm{~mL}$ of phosphate-buffered saline (PBS). The number of B. suis S2 were counted by plating on TSA. Escherichia coli strain DH5 $\alpha$ (takana) was cultured in Luria-Bertani (LB) medium. When appropriate, $50 \mu \mathrm{g} / \mathrm{mL}$ gentamicin or ampicillin were respectively added. Plasmid PUC19 was purchased from Takana.

\subsection{Construction of the Mutant Strain $\Delta V c e C$}

$\Delta \mathrm{VceC}$ was constructed as described previously [29]. Briefly, primers were designed using the sequence B. suis S2 genome and plasmid PBBR1MCS-5. The $896 \mathrm{bp} \mathrm{VceC}$ upstream fragment, $964 \mathrm{bp} \mathrm{VceC} \mathrm{downstream}$ fragment, and 751 bp gentamicin fragment were obtained in three independent PCR reactions using primer STAR Max Mix with primer pairs VceC-UF: ctgcag (Pst1) TCGGAAGCGAGCACCTGA; VceC-UR: tctaga (XbaI) GCGGATACCCTCTTACACTATAAAC; VceC-DF: gagctc (SacI) CCAAGGGAGAAACCCGCA; VceC-DR: gaattc (EcoRI) CGTGTTCACAACCGATAAGG; G-F tctaga (XbaI) TTGACATAAGCCTGTTCGGTTCGTA; G-R: gagctc (SacI) TTAGGTGGCGGTACTTGGGTCGATA. After purification by gel extraction, the three fragments were cloned into PMD19T-simple, then digested with PstI and XbaI; SacI and EcoRI; and XbaI and SacI sequentially, and then subcloned into the PstI- and EcoRI-digested PUC19 plasmid. The recombinant plasmid with the correct sequence was designated PUC19-VceC. Then this plasmid was electroporated into B. suis S2, where it is incapable of autonomous replication. The potential GntR deletion mutant was selected by plating on TSA-containing gentamicin, which was then verified by PCR with primer pairs VceC-F: CTTCTCATTGGCAAGCACTTC and VceC-R: GCATCATTCGCCGTTTCA. The mutant strain was called $\Delta$ VceC.

\subsection{Cell Infection Assay}

The process of the B. suis S2 infection assays was carried out as described previously. Briefly, GTCs were seeded in 6-well plates $\left(5 \times 10^{5}\right.$ cells per well $)$ or in 24 -well plates $\left(1 \times 10^{5}\right.$ cells per well $)$ and were infected with B. suis S2 or $\triangle \mathrm{VceC}$ at a multiplicity of infection of 100:1. After $4 \mathrm{~h}$ of incubation at $37^{\circ} \mathrm{C}$ with $5 \% \mathrm{CO}_{2}$ atmosphere, GTCs were washed three times with PBS and then further cultured with cell culture medium containing $50 \mu \mathrm{g} / \mathrm{mL}$ kanamycin to eliminate B. suis $\mathrm{S} 2$ or $\Delta \mathrm{VceC}$ adhering to the GTCs and in the culture medium. After $1 \mathrm{~h}$, the GTCs were washed three times with PBS and were further cultured with cell culture medium containing $25 \mu \mathrm{g} / \mathrm{mL}$ kanamycin to avert continuous infection. This point in time was considered $0 \mathrm{~h}$ and the time point of treatment with Tm (ER stress activator) and 4-PBA (ER stress antagonist). The cells were collected, and relevant experiments were performed at specific times $(0,6,12,24$, and $48 \mathrm{~h})$. 
For intracellular survival assays, cells were seeded in 24-well plates prior to infection. Then, cells were infected with $B$. suis $\mathrm{S} 2$ or $\triangle \mathrm{VceC}$ as described. At different times following infection, wells of infected cells were washed three times with PBS and lysed with $0.5 \%$ Triton X-100 in PBS for 10 min. The lysates were serial diluted in PBS and plated onto TSA for $72 \mathrm{~h}$ to determine the colony-forming units (CFUs).

For adherence, cells were seeded in 24-well plates prior to infection. Then, cells were infected with B. suis S2 or $\triangle \mathrm{VceC}$ as described. After $1 \mathrm{~h}$ of incubation at $37{ }^{\circ} \mathrm{C}$ with $5 \% \mathrm{CO}_{2}$ atmosphere, wells of infected cells were washed three times with PBS and lysed with $0.5 \%$ Triton X-100 in PBS for $10 \mathrm{~min}$. Next, the lysates were serial diluted in PBS and plated onto TSA for $72 \mathrm{~h}$ to determine the colony-forming units (CFUs).

\subsection{Western Blot Analysis}

GTCs were harvested in a tube after infection at $0,12,24$, and $48 \mathrm{~h}$, and then lysed on ice for $30-45 \mathrm{~min}$ in lysis buffer. The supernatant was obtained by centrifugation for $15 \mathrm{~min}$ at $14,000 \mathrm{rpm}$ at $4{ }^{\circ} \mathrm{C}$. The protein concentration was determined by the Bicinchoninic acid (BCA) assay. Total cellular protein was extracted with $5 \times$ sodium dodecyl sulfate polyacrylamide gel electrophoresis (SDS-PAGE) loading buffer after boiling for $5 \mathrm{~min}$ in water. Samples were electrophoresed on a $12 \%$ polyacrylamide gel for SDS-PAGE. The gels were then electro-transferred onto polyvinylidene fluoride (PVDF) membranes. The membranes were blocked for $1 \mathrm{~h}$ in Tris-buffered saline containing 0.5\% Tween-20 (TBST) with $5 \%-10 \%$ skimmed milk at room temperature and then incubated overnight at $4{ }^{\circ} \mathrm{C}$ in blocking solution containing GRP78 (Abcam, 1:1000 dilution), CHOP (Abcam, 1:1000 dilution), ATF-6 (Abcam, 1:1000 dilution), phosphoIRE1 (Abcam, 1:1000 dilution), phosphoeIF2 $\alpha$ (Abcam, 1:1000 dilution), caspase-3 (proteintech, 1:1000 dilution), caspase-9 (proteintech, 1:1000 dilution), and anti- $\beta$-actin (Tianjin Sungene Biotech Co, 1:1000 dilution). The membranes were washed five times with TBST for $5 \mathrm{~min}$ and then incubated for $1 \mathrm{~h}$ with the corresponding secondary antibody conjugated to horse radish peroxidase (HRP) (1:5000, Zhongshan Golden Bridge Biotechnology, Nanjing, China). Finally, the membranes were washed five times in TBST for $5 \mathrm{~min}$. The blots were visualized using the Gel Image System (Tannon, Biotech, Shanghai, China).

\subsection{Immunofluorescence Assay}

GTCs were seeded in 24-well plates and were infected with B. suis S2 and $\Delta \mathrm{VceC}$ at $100 \mathrm{MOI}$. At 0 and $24 \mathrm{~h}$ post infection, infected cells were washed twice with PBS and then fixed with $4 \%$ paraformaldehyde at room temperature for $30 \mathrm{~min}$. After three washes with PBS, cells were incubated with PBS containing $0.25 \%$ Triton X-100 at room temperature for $20 \mathrm{~min}$. After three washes with PBS, goat anti-brucella polyclonal antibody (1:100 dilution), rabbit anti-GRP78 monoclonal antibody (1:200 dilution), and mouse anti-LAMP-1 monoclonal antibody (1:200 dilution) were used as the primary antibody. Donkey anti-goat alexa fluor 555, donkey anti-mouse alexa fluor 488, and donkey anti-rabbit alexa fluor 488 were used as the secondary antibody at 1:200 dilutions. Next, coverslips were mounted on glass slides, and cells were observed under a microscope. Assays were performed in triplicate.

\subsection{Statistical Analysis}

Statistical analysis was performed using Graphpad Prism software 6 (GraphPad software Inc., La Jolla, CA, USA). Statistical significance was determined using two-way ANOVA or one-way ANOVA. $P$ values less than 0.05 were considered statistically significant.

Supplementary Materials: Supplementary materials can be found at http://www.mdpi.com/1422-0067/20/17/ 4104/s1. Supplementary Figure 1: Results of cell apoptosis after B. suis S2 and $\Delta$ VceC infection at $12 \mathrm{~h}$ and $48 \mathrm{~h}$.

Author Contributions: Formal analysis, F.B., J.L., C.X. and G.Z.; Funding acquisition, D.Z. and A.W.; Investigation, F.Z. and D.Z.; Writing-original draft, F.Z. and D.Z.; Writing—review \& editing, Y.J. and A.W. 
Acknowledgments: This research was funded by National Key R\&D Program of China (2018YFD0500900); National Natural Science Foundation of China (31672584, 31702310); China Postdoctoral Science Foundation (2016M602883); Natural Science Basic Research Plan in Shaanxi Province of China (2017JQ30100).

Conflicts of Interest: The authors declare that they have no competing interests.

\section{References}

1. Pappas, G.; Papadimitriou, P.; Akritidis, N.; Christou, L.; Tsianos, E.V. The new global map of human brucellosis. Lancet Infect. Dis. 2006, 6, 91-99. [CrossRef]

2. Byndloss, M.X.; Tsolis, R.M. Brucella spp. Virulence Factors and Immunity. Annu. Rev. Anim. Biosci. 2016, 4, 111-127. [CrossRef] [PubMed]

3. Samartino, L.E.; Enright, F.M. Pathogenesis of abortion of bovine brucellosis. Comp. Immunol. Microbiol. Infect. Dis. 1993, 16, 95-101. [CrossRef]

4. Celli, J. The changing nature of the Brucella-containing vacuole. Cell. Microbiol. 2015, 17, 951-958. [CrossRef] [PubMed]

5. Ahmed, W.; Zheng, K.; Liu, Z.F. Establishment of Chronic Infection: Brucella's Stealth Strategy. Front. Cell. Infect. Microbiol. 2016, 6, 30. [CrossRef] [PubMed]

6. Pascual, D.W.; Yang, X.; Wang, H.; Goodwin, Z.; Hoffman, C.; Clapp, B. Alternative strategies for vaccination to brucellosis. Microbes Infect. 2018, 20, 599-605. [CrossRef] [PubMed]

7. Jaiswal, V.; Chauhan, R.S.; Rout, C. Common antigens prediction in bacterial bioweapons: A perspective for vaccine design. Infect. Genet. Evol. 2014, 21, 315-319. [CrossRef] [PubMed]

8. Caporale, V.; Bonfini, B.; Di Giannatale, E.; Di Provvido, A.; Forcella, S.; Giovannini, A.; Tittarelli, M.; Scacchia, M. Efficacy of Brucella abortus vaccine strain RB51 compared to the reference vaccine Brucella abortus strain 19 in water buffalo. Vet. Ital. 2010, 46, 5-11.

9. Zhu, L.; Feng, Y.; Zhang, G.; Jiang, H.; Zhang, Z.; Wang, N.; Ding, J.; Suo, X. Brucella suis strain 2 vaccine is safe and protective against heterologous Brucella spp. infections. Vaccine 2016, 34, 395-400. [CrossRef]

10. Myeni, S.; Child, R.; Ng, T.W.; Kupko, J.J., 3rd; Wehrly, T.D.; Porcella, S.F.; Knodler, L.A.; Celli, J. Brucella modulates secretory trafficking via multiple type IV secretion effector proteins. PLoS Pathog. 2013, 9, e1003556. [CrossRef] [PubMed]

11. De Jong, M.F.; Sun, Y.H.; den Hartigh, A.B.; van Dijl, J.M.; Tsolis, R.M. Identification of VceA and VceC, two members of the $\mathrm{VjbR}$ regulon that are translocated into macrophages by the Brucella type IV secretion system. Mol. Microbiol. 2008, 70, 1378-1396. [CrossRef] [PubMed]

12. De Jong, M.F.; Starr, T.; Winter, M.G.; den Hartigh, A.B.; Child, R.; Knodler, L.A.; van Dijl, J.M.; Celli, J.; Tsolis, R.M. Sensing of Bacterial Type IV Secretion via the Unfolded Protein Response. mBio 2013, 4, e00418-12. [CrossRef] [PubMed]

13. Keestra-Gounder, A.M.; Byndloss, M.X.; Seyffert, N.; Young, B.M.; Chavez-Arroyo, A.; Tsai, A.Y.; Cevallos, S.A.; Winter, M.G.; Pham, O.H.; Tiffany, C.R.; et al. NOD1 and NOD2 signalling links ER stress with inflammation. Nature 2016, 532, 394. [CrossRef] [PubMed]

14. Von Bargen, K.; Gorvel, J.P.; Salcedo, S.P. Internal affairs: Investigating the Brucella intracellular lifestyle. FEMS Microbiol. Rev. 2012, 36, 533-562. [CrossRef] [PubMed]

15. Celli, J.; de Chastellier, C.; Franchini, D.M.; Pizarro-Cerda, J.; Moreno, E.; Gorvel, J.P. Brucella evades macrophage killing via VirB-dependent sustained interactions with the endoplasmic reticulum. J. Exp. Med. 2003, 198, 545-556. [CrossRef] [PubMed]

16. Smith, J.A.; Khan, M.; Magnani, D.D.; Harms, J.S.; Durward, M.; Radhakrishnan, G.K.; Liu, Y.P.; Splitter, G.A. Brucella induces an unfolded protein response via TcpB that supports intracellular replication in macrophages. PLoS Pathog. 2013, 9, e1003785. [CrossRef] [PubMed]

17. Wang, X.; Lin, P.; Li, Y.; Xiang, C.; Yin, Y.; Chen, Z.; Du, Y.; Zhou, D.; Jin, Y.; Wang, A. Brucella suis Vaccine Strain 2 Induces Endoplasmic Reticulum Stress that Affects Intracellular Replication in Goat Trophoblast Cells In vitro. Front. Cell. Infect. Microbiol. 2016, 6, 19. [CrossRef]

18. Cao, S.S.; Kaufman, R.J. Unfolded protein response. Curr. Biol. 2012, 22, R622-6. [CrossRef]

19. Lee, W.-S.; Yoo, W.-H.; Chae, H.-J. ER stress and autophagy. Curr. Mol. Med. 2015, 15, 735-745. [CrossRef]

20. Szegezdi, E.; Logue, S.E.; Gorman, A.M.; Samali, A. Mediators of endoplasmic reticulum stress-induced apoptosis. EMBO Rep. 2006, 7, 880-885. [CrossRef] 
21. Atluri, V.L.; Xavier, M.N.; de Jong, M.F.; den Hartigh, A.B.; Tsolis, R.M. Interactions of the human pathogenic Brucella species with their hosts. Annu. Rev. Microbiol. 2011, 65, 523-541. [CrossRef] [PubMed]

22. Gross, A.; Terraza, A.; Ouahrani-Bettache, S.; Liautard, J.P.; Dornand, J. In vitro Brucella suis infection prevents the programmed cell death of human monocytic cells. Infect. Immun. 2000, 68, 342-351. [CrossRef] [PubMed]

23. Tolomeo, M.; Di Carlo, P.; Abbadessa, V.; Titone, L.; Miceli, S.; Barbusca, E.; Cannizzo, G.; Mancuso, S.; Arista, S.; Scarlata, F. Monocyte and lymphocyte apoptosis resistance in acute and chronic brucellosis and its possible implications in clinical management. Clin. Infect. Dis. Off. Publ. Infect. Dis. Soc. Am. 2003, 36, 1533-1538. [CrossRef] [PubMed]

24. Zhong, Z.; Wang, Y.; Qiao, F.; Wang, Z.; Du, X.; Xu, J.; Zhao, J.; Qu, Q.; Dong, S.; Sun, Y.; et al. Cytotoxicity of Brucella smooth strains for macrophages is mediated by increased secretion of the type IV secretion system. Microbiology 2009, 155 Pt 10, 3392-3402. [CrossRef]

25. Yao, Y.; Lu, Q.; Hu, Z.; Yu, Y.; Chen, Q.; Wang, Q.K. A non-canonical pathway regulates ER stress signaling and blocks ER stress-induced apoptosis and heart failure. Nat. Commun. 2017, 8, 133. [CrossRef]

26. Masciarelli, S.; Fra, A.M.; Pengo, N.; Bertolotti, M.; Cenci, S.; Fagioli, C.; Ron, D.; Hendershot, L.M.; Sitia, R. $\mathrm{CHOP}$-independent apoptosis and pathway-selective induction of the UPR in developing plasma cells. Mol. Immunol. 2010, 47, 1356-1365. [CrossRef] [PubMed]

27. Martinon, F.; Chen, X.; Lee, A.H.; Glimcher, L.H. TLR activation of the transcription factor XBP1 regulates innate immune responses in macrophages. Nat. Immunol. 2010, 11, 411-418. [CrossRef]

28. Taguchi, Y.; Imaoka, K.; Kataoka, M.; Uda, A.; Nakatsu, D.; Horii-Okazaki, S.; Kunishige, R.; Kano, F.; Murata, M. Yip1A, a novel host factor for the activation of the IRE1 pathway of the unfolded protein response during Brucella infection. PLoS Pathog. 2015, 11, e1004747. [CrossRef]

29. Zhou, D.; Zhi, F.J.; Qi, M.Z.; Bai, F.R.; Zhang, G.D.; Li, J.M.; Liu, H.; Chen, H.T.; Lin, P.F.; Tang, K.Q.; et al. Brucella induces unfolded protein response and inflammatory response via GntR in alveolar macrophages. Oncotarget 2018, 9, 5184-5196. [CrossRef]

(C) 2019 by the authors. Licensee MDPI, Basel, Switzerland. This article is an open access article distributed under the terms and conditions of the Creative Commons Attribution (CC BY) license (http://creativecommons.org/licenses/by/4.0/). 